\title{
Analysis of dependence of the 2FSI subsystem parameters on the labyrinth seal diameter of the centrifugal compressor in the gas pumping unit
}

\author{
L N Butymova ${ }^{1}$ and V Y Modorskii ${ }^{1}$ \\ ${ }^{1}$ Perm National Research Polytechnic University (PNRPU), Komsomolsky Av. 29, Perm, \\ Russia, 614990
}

\begin{abstract}
The system of centrifugal compressor GTU has several 2FSI subsystems: impeller, labyrinth seals (LS), dummies, etc. The article analyzes dependence of one subsystem - the labyrinth seal - on its diameter. The effect of this parameter on the wave processes occurring in labyrinth seals during vibrations of the rotor is studied. A solution is obtained for a nonstationary aeroelastic three-dimensional gas flow problem in deformable LS. The amplitude-frequency characteristics of the gas dynamic and vibration parameters of the LS CC of GTU are obtained. Time dependences of the gas-dynamical force and the work of gasdynamic forces in the LS zone at different rotor diameters are obtained.
\end{abstract}

\section{Physical statement}

The computational model of the labyrinth seal was considered, which is a set of the LS gas-dynamic gap and the LS steel ring in a 3D statement. The change in the gas dynamic force in time, calculated through the pressure field acting directly on the rotor [1-6], was monitored. The rotor motion of the was described by the harmonic law with given amplitude and frequency [7-11]. When assigning the rotor oscillation frequency, the blade frequency and the rotational frequency were taken into account. The rotor geometric characteristics were varied and their influence on the wave processes occurring in the labyrinth seals during rotor vibrations was estimated. At the LS inlet and outlet, a differential pressure was set. Particular attention was paid to evaluation of wave processes in the circumferential direction [12-18]. As a parameter of the investigated dependences, the rotor diameter in the LS zone is adopted.

\section{The solid staging}

The solid model (basic variant) is a circular gas-dynamic gap of LS with width $37 \mathrm{~mm}$, height $500 \mu \mathrm{m}$ and diameter $422 \mathrm{~mm}$. The solid-state LS ring is a complex stepped construction (Figure 1). The construction material is steel, the pressure drop is not taken into account, the working medium is perfect gas.

The plan for carrying out the computational experiment on studying resonances in the 2FSI subsystem "labyrinth seals" (LS) of GTU is shown in Table 1. The parameters vary with respect to the "basic variant”. 
Table 1. The plan for the computational experiment.

$\left(\mathrm{BV}^{1}\right)$ - basic variant

\begin{tabular}{lccc}
\hline No of variant & $1\left(\mathrm{BV}^{1}\right)$ & 2 & 3 \\
\cline { 1 - 1 } $\mathrm{D}_{\text {rotor }}, \mathrm{mm}$ & 422 & 294 & 550
\end{tabular}

The calculation lasts 8.5 hours when examining the perfect gas and absence of pressure drop. Preliminary calculations have shown that the calculation time increases and reaches 48 hours for variants corresponding to real gases and presence of pressure drop in the LS. The amount of computational resources required is limited by the ANSYS capabilities for parallelizing the durability part of 2FSI calculations. Acceleration of calculations of the structure stress-strain state in the 2FSI statement was observed when only up to 8 cores (1 node) were used. With a further increase in the number of calculation cores, the count rate did not increase.

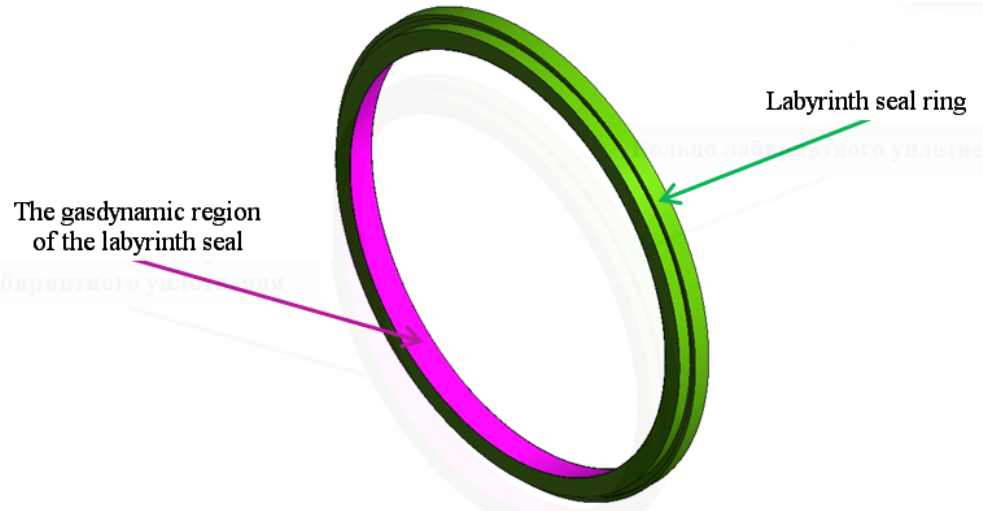

Figure 1. Solid-state model of the 2FSI subsystem of the LS CC GTU.

\section{The mesh model}

The minimum size of the finite elements of the mesh models of the LS ring and the gas-dynamic gap of the LS is $3.75 \mathrm{~mm}$, the element type is hexahedral (Figure 2). For the feasibility of 2FSI calculations in the construction of mesh models of the gas dynamic region and durability region, it was ensured that the corresponding network elements coincide at the data transfer boundary from one type of analysis to another.

a)

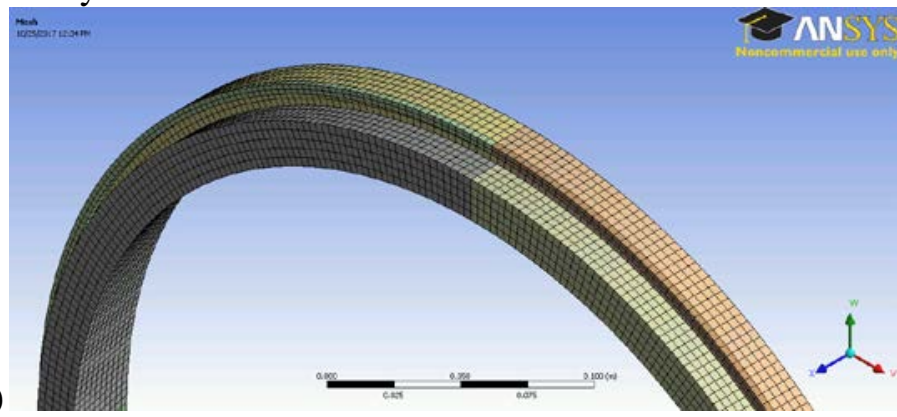

b)

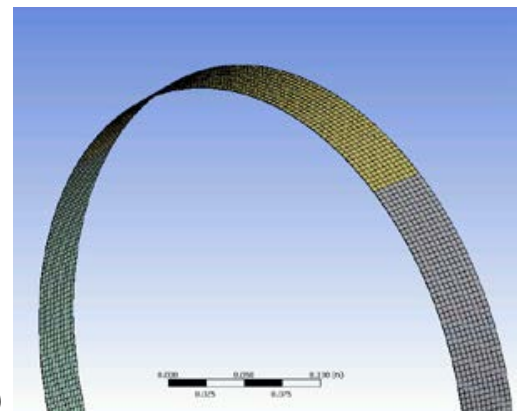

Figure 2. The mesh model of the 2FSI subsystem of the LS CC GTU: (a) - mesh model of the labyrinth seal ring; (b) - mesh model of the gas-dynamic region of the labyrinth seal

\section{Preparation and carrying out calculations on estimation of influence of LS geometrical characteristics on circumferential oscillations in 2FSI subsystem LS CC GTU}

In accordance with the plan, the influence of the LS geometric characteristics on circumferential oscillations in the 2FSI subsystem of the LS GTU is investigated. Time dependences of pressure, gas dynamic force, work of gas dynamic force and total displacements in the LS ring LU on the rotor diameter are obtained (Figure 3). 
a)
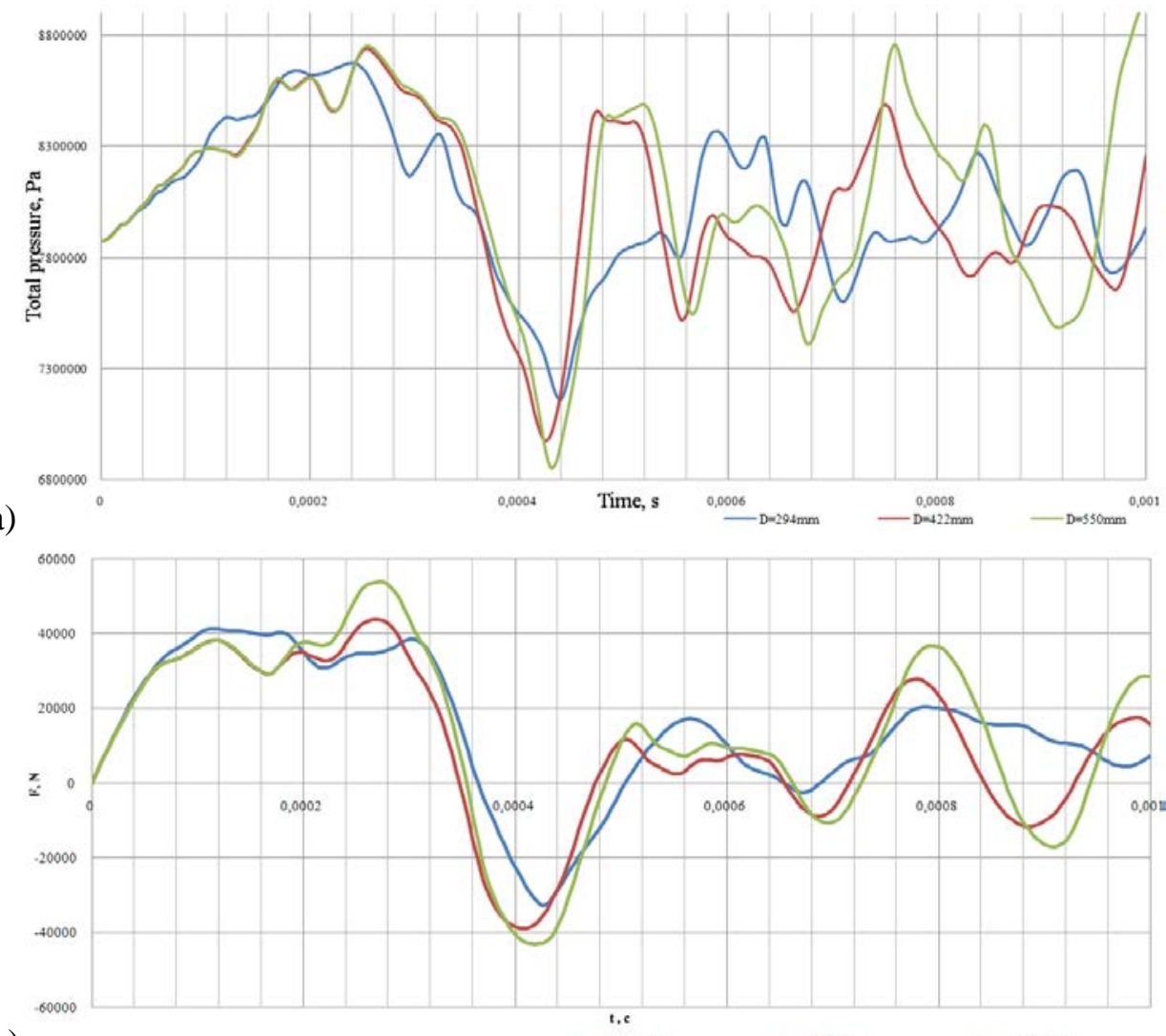

b)

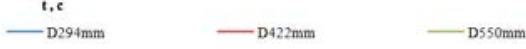

C)
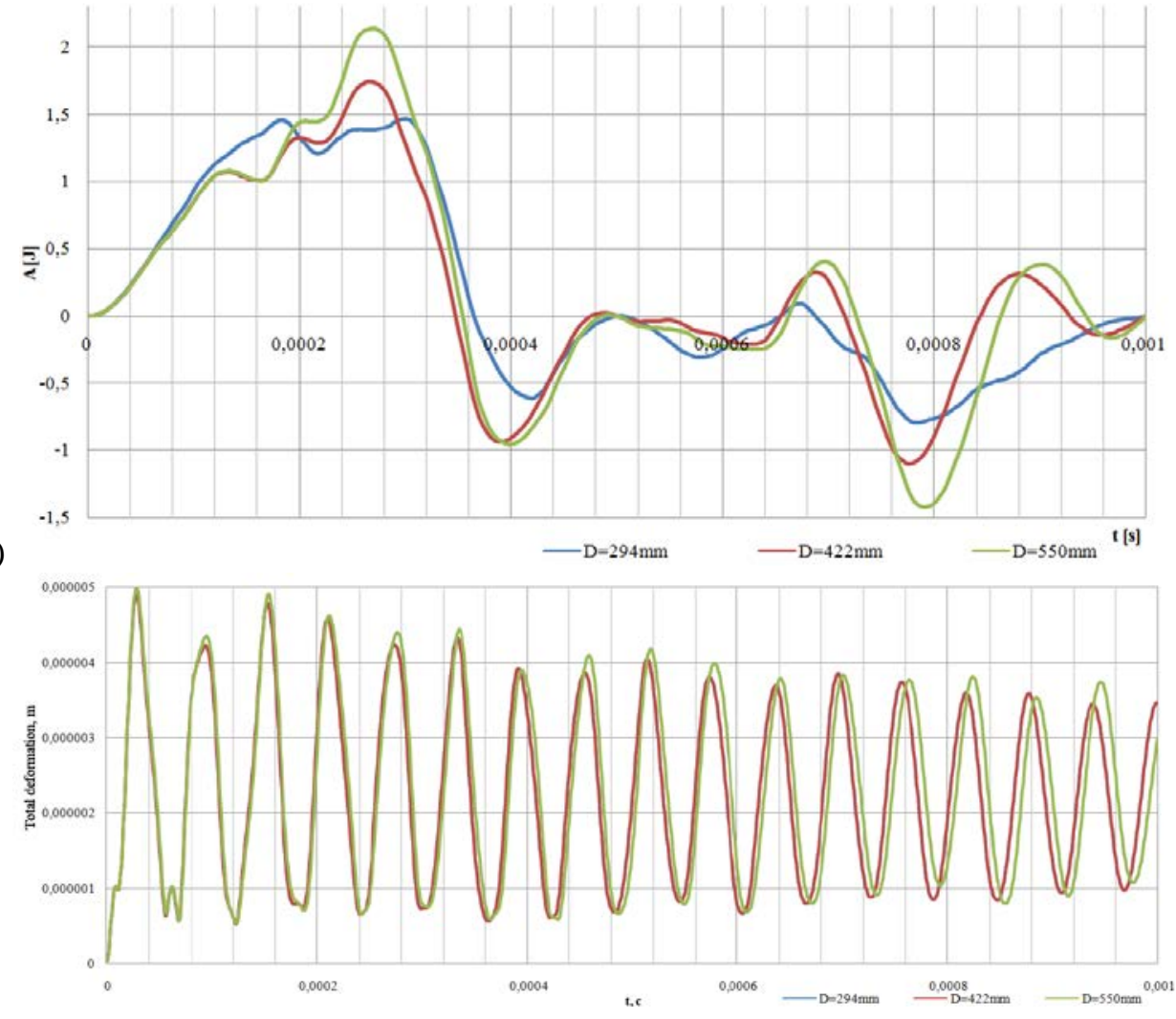

Figure 3. Time dependences of the 2FSI parameters of the LS CC GTU subsystem on the rotor diameter: (a) pressure in the LS gas-dynamic gap; (b) gas-dynamic force; (c) work of the gas dynamic force; (d) total displacements in the LS ring. 

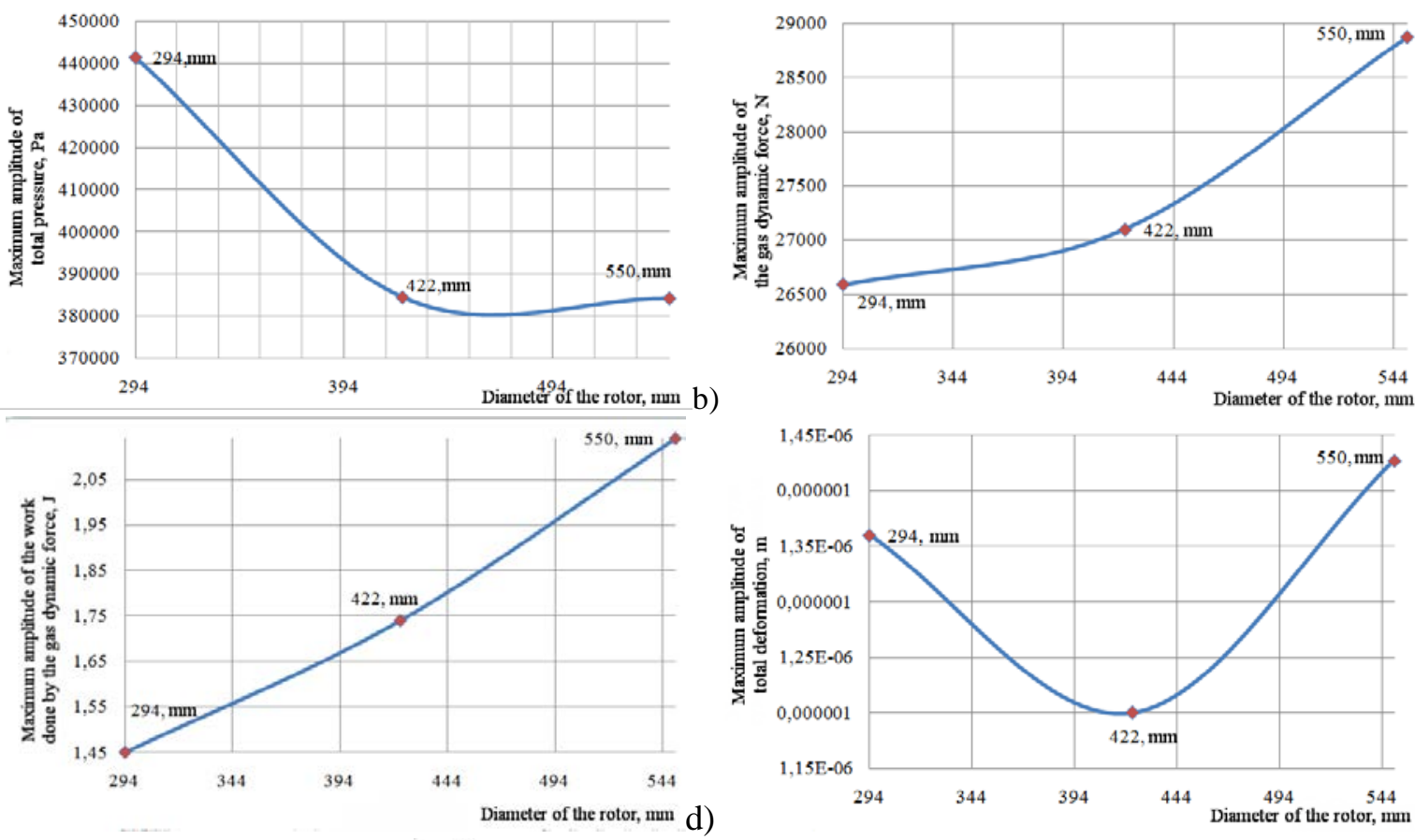

c)

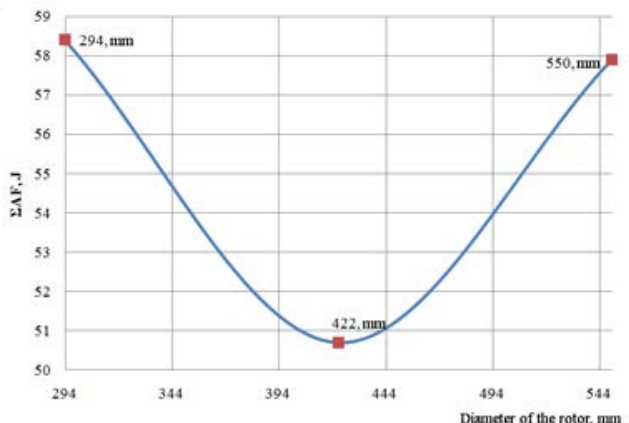

e)

Figure 4. Analysis of the time dependences of the LS subsystem 2FSI parameters CC GTU on the rotor diameter: (a) - maximum amplitude of the braking pressure; (b) - maximum amplitude of the gas dynamic force; (c) - maximum amplitude of the gas dynamic force; (d) - maximum amplitude of the total displacements; (e) - total work of the gas dynamical force.

When analyzing time dependences of the 2FSI subsystem of the LS CC GTU on the LS geometric parameters (Figure 4), it was revealed:

- with increase in the shaft diameter from $294 \mathrm{~mm}$ to $550 \mathrm{~mm}$, the maximum amplitude of the gas dynamic force is observed at the diameter of $550 \mathrm{~mm}$ and equal to $28.9 \mathrm{kN}$, the minimum value of the gas dynamic force amplitude is observed at the diameter of $294 \mathrm{~mm}$ and equal to $26.6 \mathrm{kN}$;

- the maximum amplitude frequency of the gas dynamic force is constant and equal to $1.5 \mathrm{kHz}$ for the considered range of diameters and is not equal to the frequency of the driving force $(0.95 \mathrm{kHz})$;

- with an increase in diameter from $294 \mathrm{~mm}$ to $550 \mathrm{~mm}$, the maximum amplitude of the oscillation of the braking pressure does not change;

- with increase in diameter from $294 \mathrm{~mm}$ to $550 \mathrm{~mm}$, the maximum amplitude of total displacements does not change;

- with increase in diameter from $294 \mathrm{~mm}$ to $550 \mathrm{~mm}$ (by 1.9 times), increase in the maximum amplitude of the work done by gas dynamic force from $1.45 \mathrm{~J}$ to $2.14 \mathrm{~J}$ (1.5 times) is observed;

- in figure 4a, it is seen that at the diameter of $294 \mathrm{~mm}$, the pressure oscillation amplitude is maximal. Spectral analysis showed that the diameter equal to $294 \mathrm{~mm}$ corresponds to an oscillatory process with a resonant frequency of $1800 \mathrm{~Hz}$, which is multiple of the rotor oscillation frequency (2 times). It should be clarified that the rotor oscillation frequency was set equal to the blade frequency. 
The labyrinth seals with diameters equal to $422 \mathrm{~mm}$ and $550 \mathrm{~mm}$ correspond to oscillatory processes with resonant frequencies of $1260 \mathrm{~Hz}$ and $720 \mathrm{~Hz}$, respectively, which are not multiple of the exciting frequency. In this case, the pressure oscillation amplitude at these diameters is reduced (Figure 4a.)

\section{Conclusions}

1. The influence of the DN diameter on the character of the oscillatory process in the LS is revealed.

2. If the frequencies of oscillations of the rotor and gas coincide (in the circumferential direction), the maximum amplitude of the braking pressure in the LS is observed.

3. The total work of gas-dynamic forces is positive in the considered range of diameters, which indicates the possibility of "build-up" of the system (Figure 4e).

4. To reduce the amplitude of the oscillatory process, it is necessary to "detune" from the coincidence of the oscillation frequencies of the rotor and the gas dynamic volume of the LS by changing the diameter of the LS at the design stage.

\section{References}

[1] Butymova L N, Modorskii V Ya and Petrov V Yu 2016 Numerical modeling of interaction in the dynamic system "gas-structure" with harmonic motion of the piston in the variable section pipe ICMAR AIP Conference Proceedings 1770030103

[2] Butymova L N and Modorskii V Ya 2016 One-way FSI simulation of the phase and the geometric parameters of the model of compressor blades on the oscillating gas-dynamic processes pipe MATEC Web Conf. 7504007

[3] Mekhonoshina E V and Modorskii V Ya 2016 Impact of magnetic suspension stiffness on aeroelastic compressor rotor vibrations of gas pumping units ICMAR AIP Conference Proceedings $\mathbf{1 7 7 0} 030113$

[4] Makarov A A and Zaitsev N N 2015 Engineering and theoretical problems of using labyrinth seals in high-speed rotary machines Bulletin of PNRPU. Aerospace engineering 42 61-81 (in Russian)

[5] Butymova L N and Modorskii V Ya 2017 Development and application of a unified algorithm for solving the interdisciplinary problem of modeling aeroelastic processes in the labyrinth seal of centrifugal compressors AIP Conference Proceedings 1893030067

[6] Modorskii V Y and Shevelev N A 2016 Research of aerohydrodynamic and aeroelastic processes on PNRPU HPC system ICMAR AIP Conference Proceedings 1770020001

[7] Akzholov M Zh, Alabuzhev P M, Aliev A V, Artistsheva L M and Boyko L G 1995 Numerical investigation of actual problems of mechanical engineering and mechanics of continuous and granular media by the method of large particles. Investigation of actual problems of mechanics and machine building (Moscow: National Academy of Applied Sciences, International Association of Developers and Users of the Large-Particle Method) p 1658

[8] Mekhonoshina E V and Modorskii V Y 2015 On a phase-shift of waves at the middle interface Computer Optics 39 385-391

[9] Butymova L N and Modorskii V Ya 2017 Numerical modeling of the labyrinth seal taking into account vibrations of the gas transmittal unit rotor in aeroelastic formulation CEUR Workshop Proceedings 1902 10-17

[10] di Mare L, Imregun M, Green J S and Sayma A I 2010 A numerical study of labyrinth seal flutter Journal of tribology-transactions of the ASME 132(2) 022201

[11] Zhang H, Jia XY, Pan XJ, Jiang B and Zheng Q 2016 Interaction between rotor and annular seals: interlaced and straight-through labyrinth seals Journal of propulsion and power 32(6) 1483-1493

[12] Vannini G, Bertoneri M, Nielsen K K, Iudiciani P and Stronach R 2016 Experimental results and computational fluid dynamics simulations of labyrinth and pocket damper seals for wet gas compression Journal of engineering for gas turbines and power-transactions of the ASME 138(5) 052501 
[13] Dairien A, Thouverez F, Blanc L, Helies P and Dehouve J Fluid-structure interaction in a labyrinth gas seal coupled to a flexible stator Topics in Modal Analysis \& Testing 10 1-9

[14] Zhang E J, Jiao Y H, Chen Z B, Mo W C and Wang S Parametric influences on the nonlinear dynamic responses of a rotor-bearing-foundation-labyrinth seal system Proceedings of the ASME international mechanical engineering congress and exposition 4a UNSP V04AT05A032

[15] A V Srinivasan, R A Arnoldi and A J Dennis 1984 Aeroelastic instabilities in labyrinth air seal systems Mechanical Engineering 106(6) 91-96

[16] D R Abbott 1981 Advances in labyrinth seal aeroelastic instability prediction and prevention Journal of Engineering for Power-Transactions of the ASME 103(2) 308-312

[17] Phibel R and di Mare L 2012 Comparison between a CFD code and a three control-volume model for labyrinth seal flutter predictions Proceedings of the ASME Turbo Expo 6 597-606

[18] Phibel R, di Mare L, Green J S and Imregun M 2009 Numerical investigation of labyrinth seal aeroelastic stability Proceedings of the ASME Turbo Expo 6 573-583

\section{Acknowledgments}

This study was supported by the Russian Science Foundation, project No 14-19-00877. 